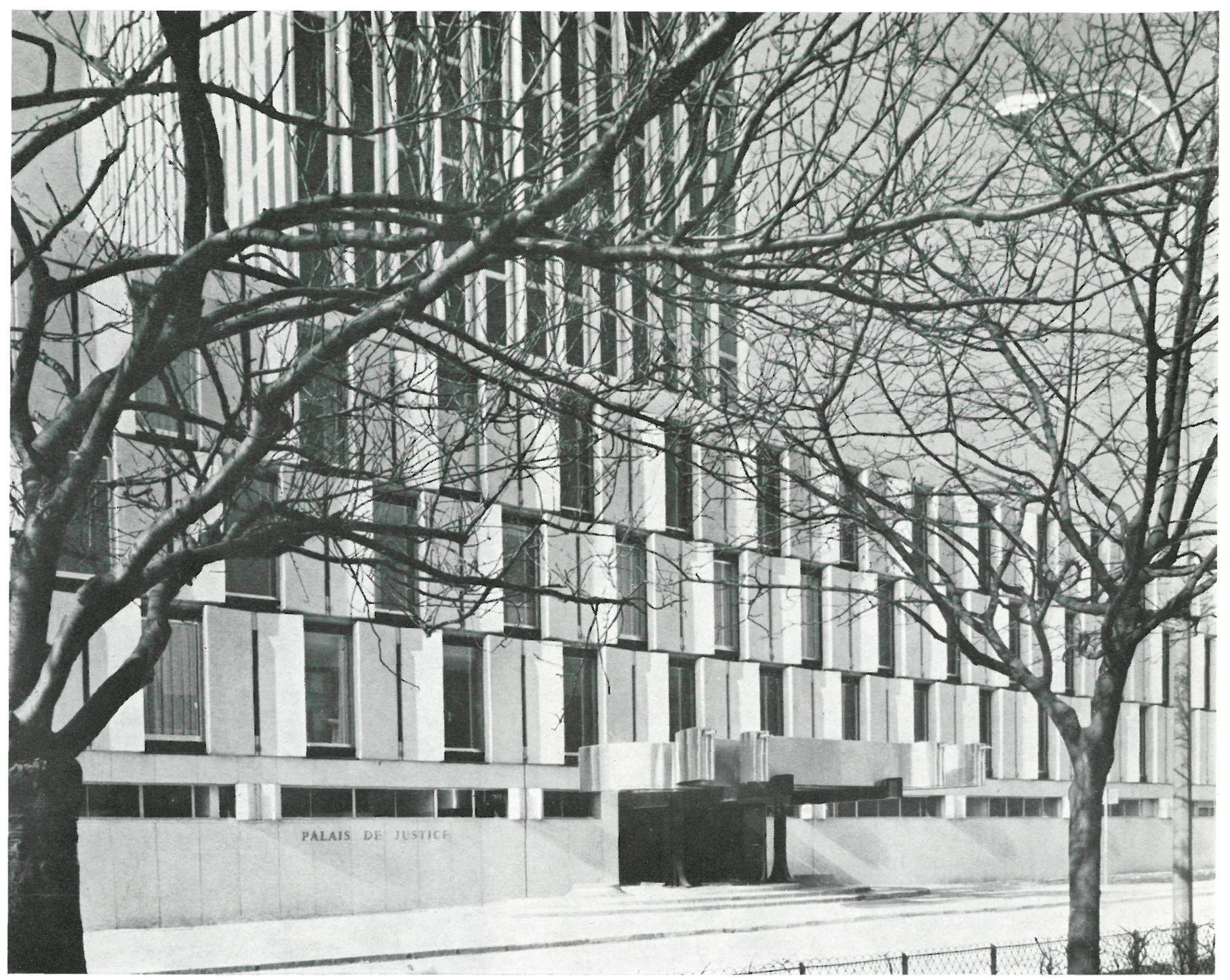

\title{
palacio de justicia de Lille - Francia
}

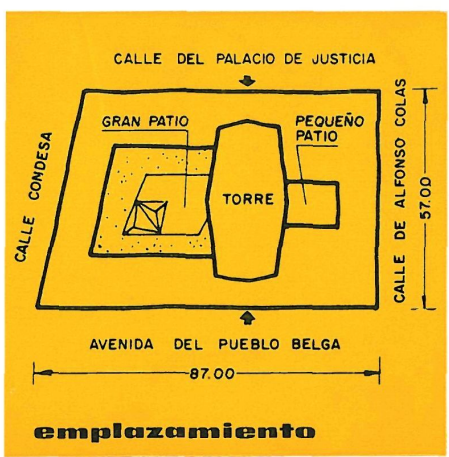

J. WILLERVAL, M. SPENDER, P. RIGNOLS, arquitectos

J. M. HERENG, ingeniero consejero

$145-77$

\section{sinopsis}

Se describe el conjunto total y después se especifican las partes del edificio del Palacio de Justicia, señalando las dependencias que contiene y la función de cada una.

En una segunda parte del artículo se comenta técnicamente la estructura de la obra, también por partes y según su nivel, dando, a la vez, detalles arquitectónicos y estéticos.

El conjunto edificado reúne características específicas formales y estructurales que lo destacan y hacen digno de especial atención y mención. 


\section{Precén numbo}

La imponente noción de la Justicia de otros tiempos se ha transformado sensiblemente a lo largo de los últimos decenios para dar lugar a un orden administrativo adaptado a la evolución de la civilización moderna, pareciéndose más a la noción de clínica del mal social que a la de los antiguos templos de Themis.

Ante la insuficiencia de su viejo Palacio de Justicia, la ciudad de Lille -en el Norté de Francia, capital industrial de Flandres, región muy poblada y laboriosa- convocó en 1958 un concurso de Arquitectura para la construcción de un nuevo conjunto que englobara el Palacio de Justicia y dependencias auxiliares, constituyendo, en cierto modo, una verdadera Ciudad Judicial.

El equipo de Jean Willerval fue el que se adjudicó la realización del proyecto, que aquí presentamos. El éxito arquitectónico de este conjunto es el fruto de una estrecha y fructífera colaboración entre el arquitecto y el ingeniero, facilitada por el hecho de que Jean Willerval y J. M. Hereng son grandes amigos de hace tiempo.

\section{Vister de comjunto}

El nuevo Palacio de Justicia está situado en un viejo distrito pintoresco de la ciudad, entre el Hospicio de la Condesa, obra histórica, la basílica de la Treille y el Conservatorio. El conjunto limita al O. con la calle del Palacio de Justicia; al S. con la calle de la Condesa; el E. con la avenida del Pueblo Belga, y al N. con la avenida de Alfonso Colas.

El conjunto de la obra presenta dos cuerpos de edificio:

Uno bajo, que circunscribe la totalidad del solar alrededor de un gran patio ajardinado.

Otro alto, la «Torre Administrativa», que domina el conjunto.

Todo ello se articula alrededor de una zona de comunicación realizada por la superposición de dos salas de «pasos perdidos», que atraviesan la obra de parte a parte y en las que concurren todas las circulaciones verticales.

La superficie construida tiene la forma de un trapecio rectángulo, cuya lado mayor —el que recae a la avenida del Pueblo Belga - tiene $85 \mathrm{~m}$ de longitud, y una profundidad de $57 \mathrm{~m}$. La superficie del solar es de $4.580 \mathrm{~m}^{2}$.

Las dos entradas principales están en el eje de la sala de «pasos perdidos» que atraviesa el conjunto del Palacio, entre la avenida del Pueblo Belga y la calle del Palacio de Justicia.

Diversas entradas secundarias abastecen a los servicios privados en las otras dos fachadas y, especialmente, la en trada al estacionamiento subterráneo.
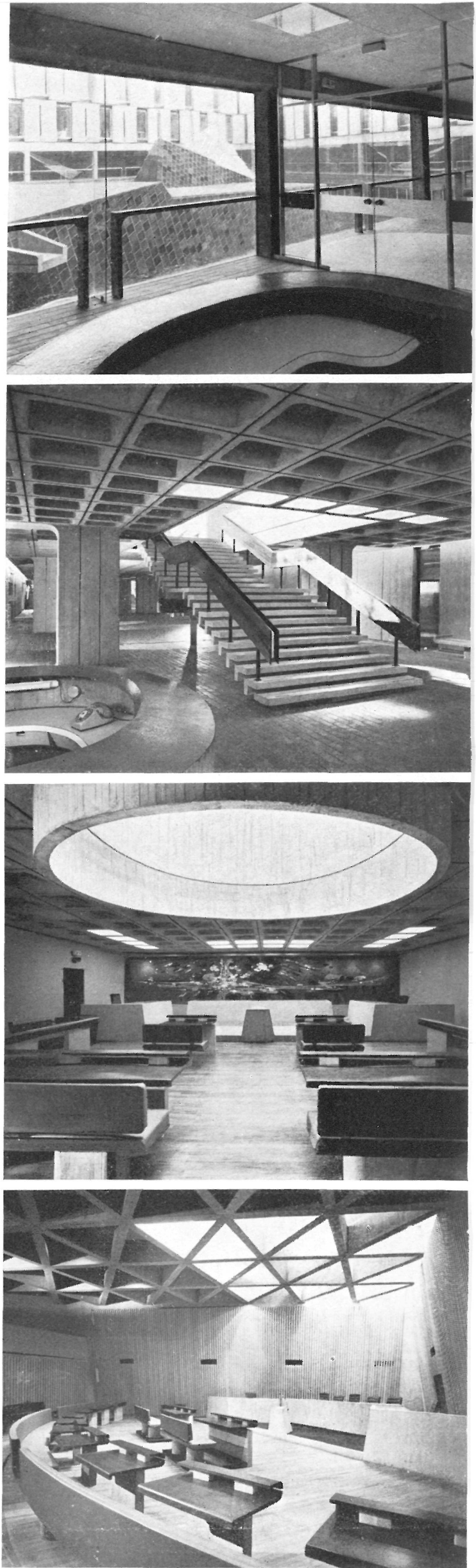


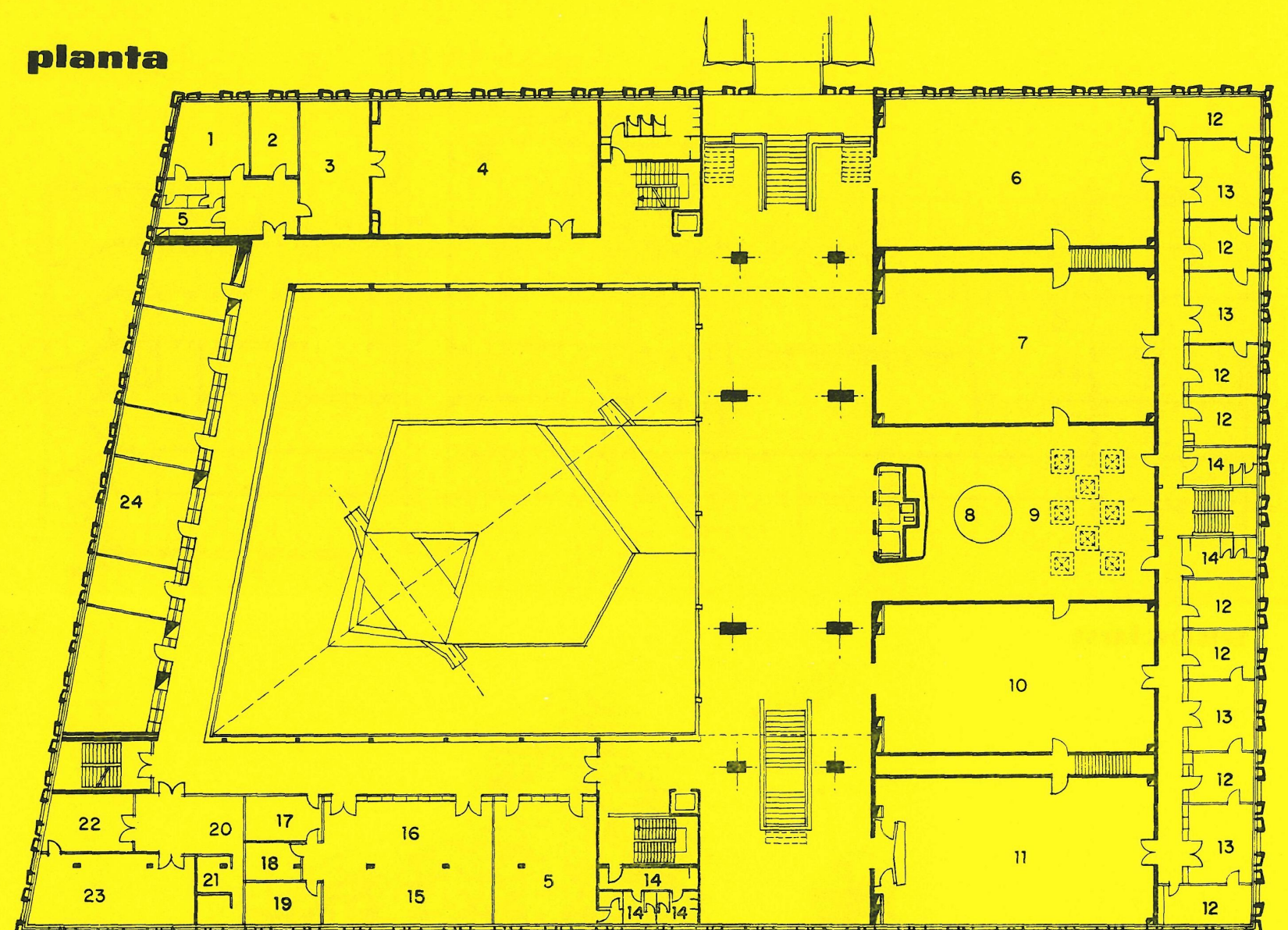

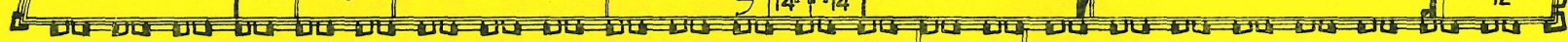

1. Presidente. -2 . Jueces. -3 . Sala de deliberaciones. -4 . Tribunal de comercio

5. Vestuario.-6. Cuarta sala.-7. Tercera sala.-8. Vacío de iluminación.-

9. Testigos.-10. Segunda sala.-11. Primera sala.-12. Vicepresidente.18. Catálo - 19. Bibloteorio - B cho del decano.-23. Sala de consejo - 24. Escribaní.

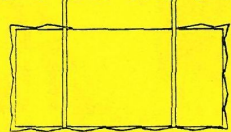

$012345 \quad 10 \quad 15 \mathrm{~m}$

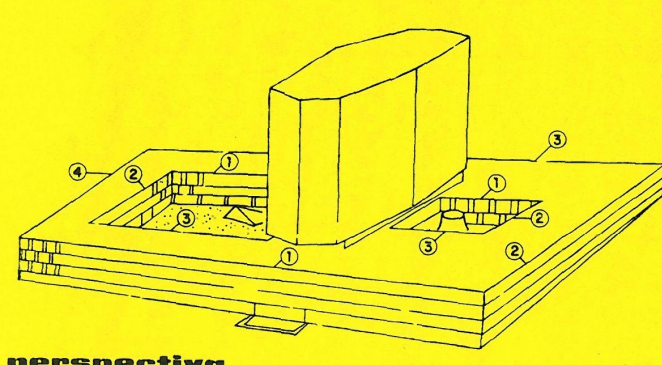

poerspectiven

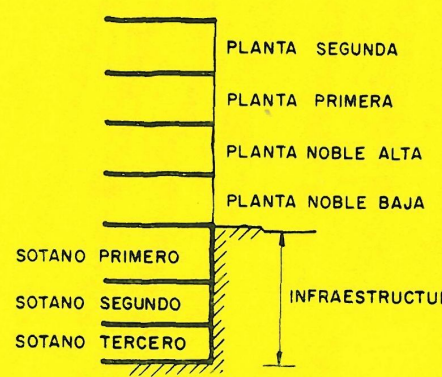

sccción Bedifficio bajo

SEcción

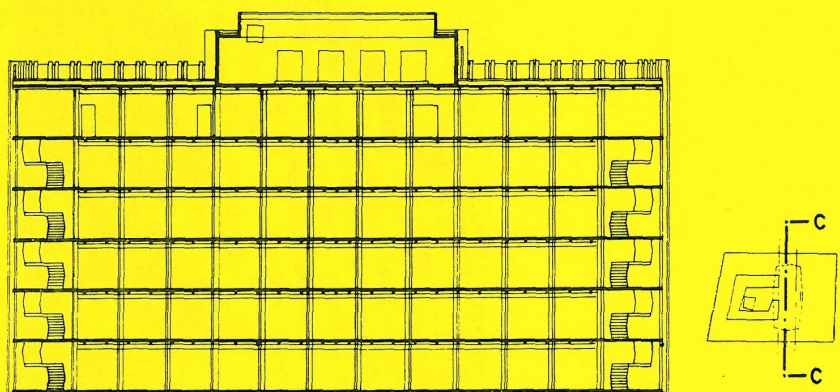



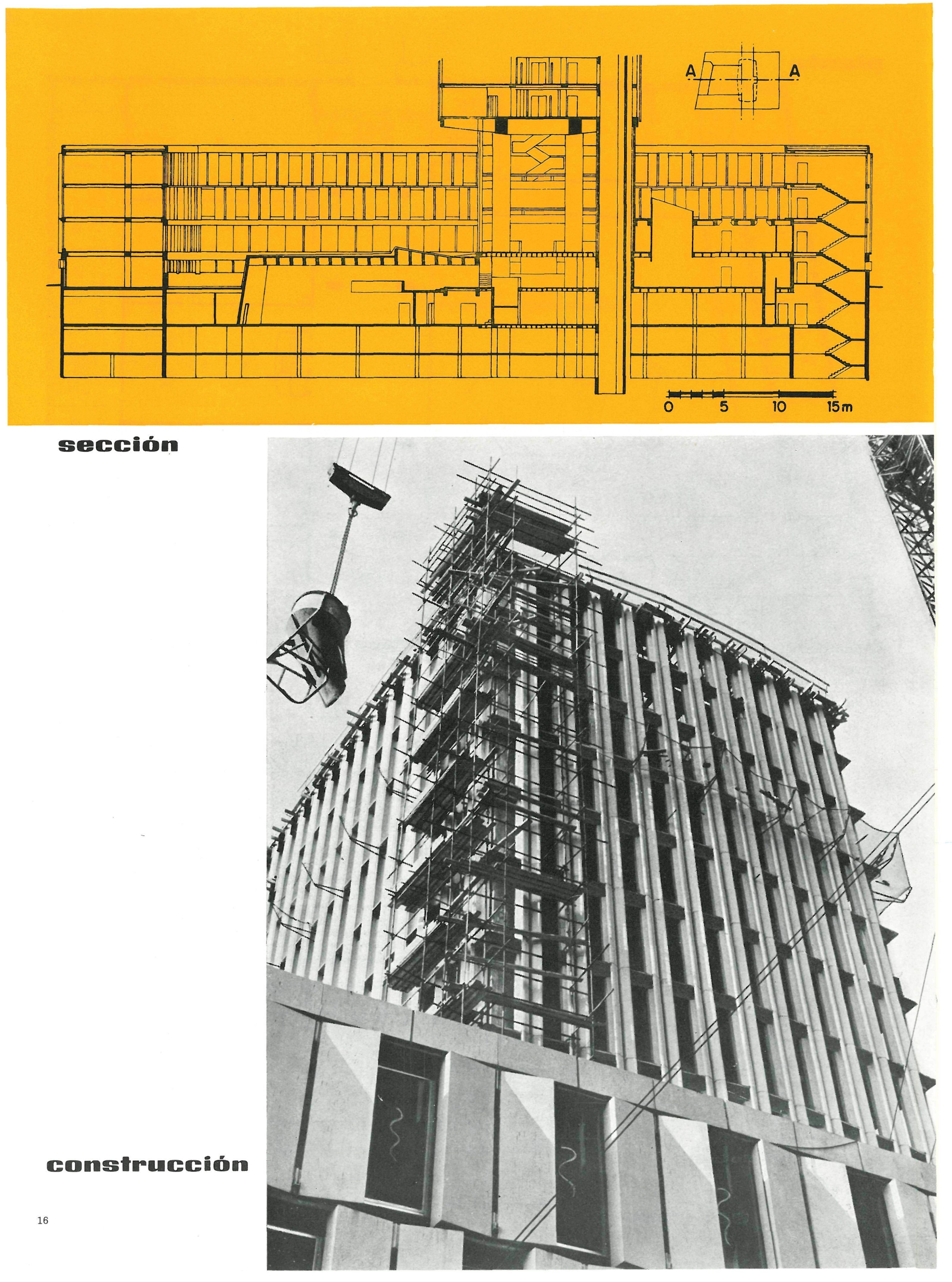


\section{Los edificios bajos}

Este conjunto ocupa la totalidad del solar en el subsuelo: tres niveles de infraestructura. En la super. estructura hay cuatro plantas que se desarrollan en cuatro fachadas exteriores continuas, sobre las calles que delimitan el edificio, y seis fachadas interiores: tres sobre el gran patio y tres sobre el pequeño, según el esquema adjunto.

La distribución es la siguiente:

- Sótano 3. (nivel -8,70): Archivos y otros servicios técnicos.

- Sótano 2. (nivel -6,30): Garajes y locales de archivos.

- Sótano 1. (nivel - 3,50): Gran sala de Instancia y sus anejos: salas de encuestas, salas de testigos, salas de consejo de familia, de deliberaciones, etc.

La rampa de salida del garaje, locales técnicos del acondicionamiento de aire. Locales para «cuerpos de delito» pesados y embarazosos. Transformador. Grupo electrógeno de socorro. Un garaje para 60 plazas y un estacionamiento para coches de policía.

Por otra parte, y a petición de la Comisión local de Monumentos Históricos, hay un enclave de $11 \times 13 \mathrm{~m}$ aproximadamente, que tiene la misión de dar acceso a los cimientos del presbiterio de la antigua Colegiata romana de San Pedro, descubiertos durante las excavaciones.

El desnivel entre la entrada de la avenida del Pueblo Belga y la de la calle del Palacio de Justicia ha permitido realizar una doble planta noble:

- Planta noble baja que comprende: del lado del patio grande, el tribunal de Instancia, los despachos de los jueces de este tribunal, los secretarios, los de los empleados, las salas de espera, los despachos de noticias, los archivos de Instancia, los guardarropas y los aseos, las cabinas telefónicas, etc. Del lado del patio pequeño, un segundo tribunal de Instancia, una sala para cuerpos de delito, salas de conferencia y de exámenes periciales, una zona aislada para estancia, cárceles y celdas, locutorios, enfermería, cocinas, etc., y tres viviendas de funcionarios.

Entre estas dos zonas, la sala baja de "pasos perdidos» que las domina y manda.

- Una planta baja superior, reservada en su mayor parte a la jurisdicción comercial: sala del tribunal de comercio de $15,5 \times 9 \mathrm{~m}$, los despachos del presidente y del juez, la sala de deliberaciones, el registro de comercio, el secretario, mecanografía, etc. El resto de este nivel está ocupado, al norte, por la jurisdicción correccional y civil, comprendiendo: la 1.a $2 .^{\mathrm{a}}$, 3. ${ }^{\mathrm{a}}$ y $4 .^{\mathrm{a}}$ estancias, todas semejantes, de $19 \times 10 \mathrm{~m}$, y una sala de testigos de $19 \times 12 \mathrm{~m}$, que da sobre el patio pequeño. Estas piezas solemnes tienen iluminación natural y su altura ocupa tres niveles.

La sala de "pasos perdidos» superior comprende estas estancias y la sala de testigos, así como sus anexos: biblioteca, salas de trabajo y de recepción, guardarropa de abogados, gabinete y sala del consejo del Decano del Colegio de Abogados, despachos y salas de deliberaciones de los presidentes y vicepresidentes. Esta sala de espera superior forma un hall inmenso y un gran centro atractivo del edificio.

- La planta primera alberga el tribunal de menores y sus servicios. En una palabra, la administración social.

- La planta segunda comprende: once despachos de jueces, despachos independientes del tribunal de menores, la sala de audiencia de la Seguridad Social y sus anexos.

\section{El edificio alto}

Este edificio descansa solamente sobre ocho pilares que atraviesan el edificio bajo por encima de la gran sala de «pasos perdidos». Se compone de nueve niveles y una planta de instalaciones en la terraza, que se distribuyen de la siguiente manera:

1. planta: despachos de jueces, salas de comisiones, servicios diversos de asistencia social.

2. ${ }^{a}$ y $3 .^{a}$ plantas: sala del tribunal, despachos del Procurador de la República, despachos de los procuradores adjuntos, sustitutos, anexos, etc.

4. ${ }^{\mathrm{a}}$ planta: la instrucción: jueces y secretarios.

5. ${ }^{\text { }}$ planta: Secretaría civil: cámara de alquileres, pensiones, adjudicaciones, accidentes de trabajo, laboratorios, etc.

6. ${ }^{a}$ planta: Secretaría correccional: hojas penales y servicios anexos.

7. planta: Archivos diversos.

8. planta: Servicio postpenal.

9. planta: Recepción.

Planta de instalaciones en la terraza: calefacción alimentada por combustible de gas natural, máquinas de ascensores, etc. 


\section{Constarageción}

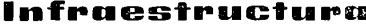

El terreno, que está constituido, de una parte, por una zona calcárea bastante compacta, y de otra parte, por una zona de margas de densidad variable, ha requerido el empleo de dos tipos de cimientos: en el primer caso, el apoyo de las obras se ha asegurado por zapatas y correas que transmiten al suelo esfuerzos que varían entre 2 y $5 \mathrm{kp} / \mathrm{cm}^{2}$, y en el segundo caso se ha conseguido el apoyo perfecto por medio de 268 pilotes Franki clavados en el suelo para evitar movimientos en los inmuebles adyacentes.

La dificultad inesperada motivada por la obligación de aislar los vestigios de la Colegiata de San Pedro, ha exigido la creación de un sisterna de apoyo particular.

Los tres niveles de los sótanos se han construido a base de muros de hormigón armado, pilares y forjados fabricados in situ con encofrados industrializados.

\section{Supperestructuras \\ Edificio bajo}

Pilares, muros interiores y forjados, en hormigón armadio fabricado in situ con encofrados industrializados. Las fachadas exteriores sobre la calle y las fachadas interiores de los patios están realizadas con la ayuda de elementos prefabricados en taller, concebidos por Hereng Ingeniería, S. A. (H.I.S.A.) y realizados por Schokbeton en Holanda. Estos elementos son parte integrante de la estructura. Su disposición en «tablero de ajedrez» y sus facetas inclinadas horizontalmente crean un armonioso juego de luces y dan vida a las fachadas. La carpintería es de aluminio estirado por presión y anodizado y la vidriera utilizada es de cristales Triplex.

\section{La gran sala de Instancia}

Bajo el gran patio está iluminada por dos lucernarios de hormigón armado visto, formados por la inter. sección de dos elementos piramidales soportados por un techo de nervios cruzados tridireccionales. La parte exterior de estos lucernarios y la parte de cubierta de esta sala de Instancia que surge entre la vegetación del gran patio, lleva revestimiento a base de cerámica barnizada y coloreada en forma de escamas.

\section{La pequeña sala de Instancia}

Tiene acceso desde el gran hall de la sala de «pasos perdidos» y se ilumina por medio de un lucernario troncocónico de hormigón armado visto. El techo es, igualmente, de nervios cruzados cuadrangulares, aunque, en este caso, los nervios no atraviesan el lucernario.

\section{Sala de "pasos perdialos" y edificio auto}

La gran sala de "pasos perdidos», de $55 \times 12 \mathrm{~m}$, va enteramente acristalada en toda su altura del lado del gran patio que la ilumina. En el lado opuesto, un altorrelieve estructurado, debido al escultor canadiense Philippe Scrive, de Quebec, proporciona juegos de sombras y de luces de bello efecto arquitectónico. Este altorrelieve se ha realizado con la ayuda de 200 elementos de hormigón premoldeados de forma tetraédrica, cuyo peso varía entre 400 y $2.800 \mathrm{kp}$ por pieza.

Para su anclaje en un muro de casi $11 \mathrm{~m}$ de longitud, ha sido preciso realizar una estructura mixta de acero y hormigón (perfiles HN de ala ancha y hormigón armado).

El techo de esta sala de "pasos perdidos» está constituido por una impresionante superficie de hormigón pretensado visto. Su forma es la de una losa octogonal muy alargada con una doble espina dorsal formada por dos importantes vigas maestras paralelas a lo largo del pliegue, y una red de vigas en voladizo en $\mathrm{X}$ que se apoyan sobre esta doble espina dorsal.

Esta superficie descansa en su parte central sobre cuatro pilares de $180 \times 80 \mathrm{~cm}$, espaciados longitudinalmente de 16 en $16 \mathrm{~m}$ y transversalmente cada $7,2 \mathrm{~m}$ y en las dos extremidades, sobre dos veces $2 \mathrm{pi}$ lares de $120 \times 80 \mathrm{~cm}$

Dominando los edificios bajos, la «torre administrativa» puede ser comparada a una construcción sobre pilotes.

La estructura propiamente dicha de la torre, encima de la superficie soporte, es del tipo reforzado: doble fila de pilares reforzados por estructuras rigidizadoras en la zona central, y forjados nervados fabricados in situ con encofrados industrializados. Las fachadas son portantes y han sido realizadas con la ayuda de pilaretes dobles con sección en forma de X, prefabricados, como en las fachadas del edificio bajo. La gran caja de ascensores en hormigón armado, realizada con la ayuda de encofrados deslizantes, completa la estabilidad de esta obra. 


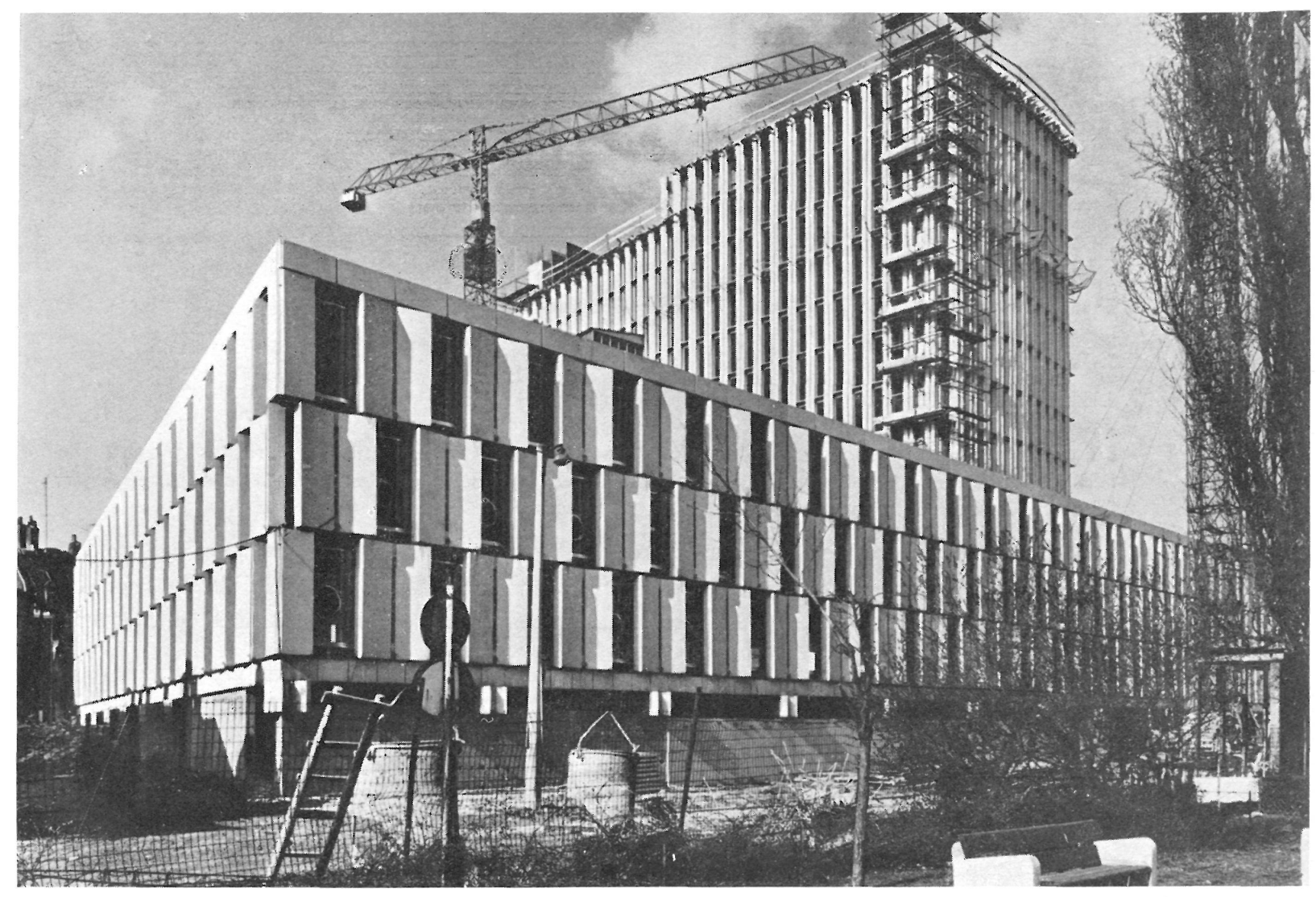

Las carpinterías son igualmente de aluminio estirado por presión y anodizado, y la vidriería de las ventanas basculantes está hecha con cristales Parsol. El antepecho, de $1 \mathrm{~m}$, tiene vidrio Securit.

Es interesante anotar que el conjunto de todos los estudios de las estructuras de esta obra ha sido realizado en España, en las oficinas barcelonesas de Hereng Ingeniería, S. A.
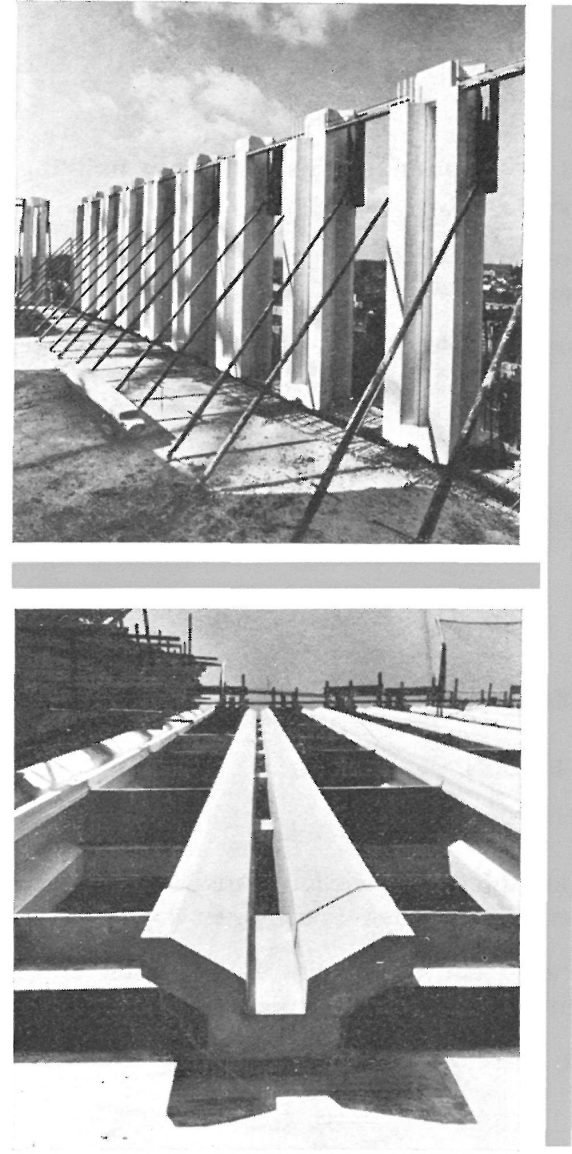

Fotos: GILLES EHRMANN
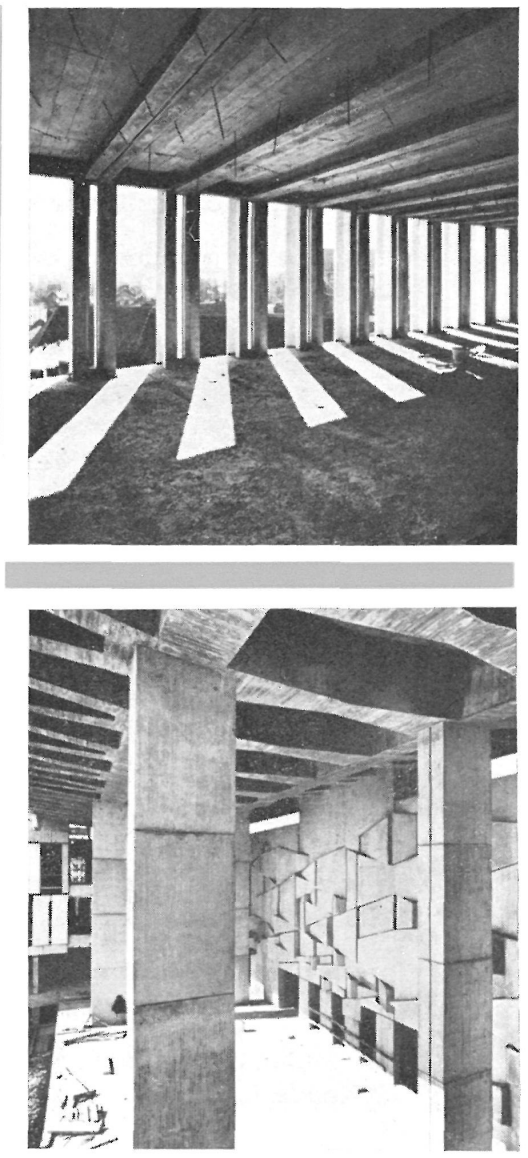


\section{Palais de Justice de Lidle = France}

J. Willerval, M. Spender et $\mathbf{P}$. Rignols, architectes

J. M. Hereng, ingénieur-conseil

La première partie de cet article décrit l'ensemble total et spécifie ensuite les parties de l'édifice du Palais de Justice tout en signalant les dépendances dont il est constitué et la fonction à laquelle chacune d'entre elles est destinée.

La deuxième partie commente techniquement la structure de l'ouvrage, également par parties et suivant son niveau, fournissant à la fois des détails esthétiques et architecturaux.

L'ensemble bâti réunit des caractéristiques spécifiques formelles et structurales qui le font remarquable et digne d'une attention et mention spéciales.

\section{Palace of Justice of Lille, Framce}

J. Willerval, M. Spender and P. Rignols, architects

J. M. Hereng, consulting engineer

The article provides a general description of this important building, and also a more detailed discussion of particular aspects of it.

There is also a technical commentary of the structure, both in its general and particular nature, and the architectural as well as aesthetic aspects if it are considered.

The whole design has formal and structural features that are unusual and noteworthy.

\section{Justizpalast in Lille - Framkreich}

J. Willerval, M. Spender et P. Rignols, Architekten

J. M. Hereng, Beratender Ingenieur

Es wird der gesamte Gebäudekomplex beschrieben. Anschliessend werden die verschiedenen Teile des Gebäudes und ihre entsprechenden Funktionen aufgeführt und spezifiziert.

Im zweiten Teil des Artikels wird der technische Aufbau der einzelnen Gebäudeteile unter Hinweis auf architektonische und ästhetische Details beschrieben.

Der Gebäudekomplex vereinigt aufsehenerregende und interessante formale und strukturelle Merkmale in sich. 